\title{
Correction to: Allogeneic Hematopoietic Cell Transplantation in Patients with Primary Immunodeficiencies in Korea: Eleven-Year Experience in a Single Center
}

\author{
Eun Sang $\mathrm{Yi}^{1,2} \cdot$ Young Bae Choi ${ }^{3} \cdot \mathrm{Na} \mathrm{Hee} \mathrm{Lee}^{4} \cdot \mathrm{Ji}_{\text {Won Lee }}{ }^{1} \cdot \mathrm{Ki}_{\text {Woong Sung }}{ }^{1} \cdot \mathrm{Hong}_{\mathrm{Hoe}} \mathrm{Koo}^{1} \cdot$ Eun-Suk Kang $^{5}$. \\ Yae-Jean $\mathrm{Kim}^{6}$ • Keon Hee Yoo ${ }^{1,7,8}$
}

Published online: 10 October 2018

(C) Springer Science+Business Media, LLC, part of Springer Nature 2018

Correction to: Journal of Clinical Immunology (2018) https://doi.org/10.1007/s10875-018-0542-7

The original version of this article unfortunately contained a mistake in the 7th author's given name.

The correct version is presented above.

The online version of the original article can be found at https://doi.org/ 10.1007/s10875-018-0542-7

Keon Hee Yoo

hema2170@skku.edu

1 Division of Hematology and Oncology, Department of Pediatrics, Samsung Medical Center, Sungkyunkwan University School of Medicine, Seoul, South Korea

2 Department of Pediatrics, Korea University Guro Hospital, Korea University College of Medicine, Seoul, South Korea

3 Department of Pediatrics, Chungbuk National University Hospital, Cheongju, South Korea

4 Department of Pediatrics, Cha Bundang Medical Centre, Cha University, Seongnam, South Korea
Departments of laboratory Medicine \& Genetics, Samsung Medical Center, Sungkyunkwan University School of Medicine, Seoul, South Korea

6 Division of Pediatric Infectious Diseases and Immunodeficiency, Department of Pediatrics, Samsung Medical Center, Sungkyunkwan University School of Medicine, Seoul, South Korea

Department of Health Science and Technology, SAIHST, Sungkyunkwan University, Seoul, South Korea

8 Stem Cell \& Regenerative Medicine Institute, Samsung Medical Center, Seoul, South Korea 